\title{
DIVERSIDAD DEL ZOOPLANCTON EN LA RESERVA NACIONAL DE JUNÍN, PERÚ
}

\section{ZOOPLANKTON DIVERSITY FROM THE JUNIN NATURAL RESERVE, PERU}

\author{
José Iannacone $e^{1,2}$ y Lorena Alvariño ${ }^{1}$
}

\begin{abstract}
Resumen
Se evaluó el zooplancton en cuatro de sus localidades de la Reserva Nacional de Junín, Perú: Puente y Represa de Upamayo, Pari y Óndores. Se calculó para las comunidades zooplanctónicas la diversidad alfa y beta. El zooplancton capturado fue adjudicado a gremios alimentarios. Se encontró un total de 35 taxas zooplantónicos. El orden descendente con relación a la riqueza de especies acuáticas fue Pari > Represa de Upamayo = Puente de Upamayo > Óndores. Los cladóceros fueron más abundantes y con mayor riqueza de especies en la localidad de Pari. Seis especies fueron compartidas por las cuatro localidades: Brachionus dimidiatus Bryce 1931 (Rotifera), Rhabdolaimus terrestris de Mann 1880 (Nematoda), Echiniscus sp. (Tardigrada), Aelosoma variegatum Vejdovsky 1866 (Annelida), una especie de calanoideo (Artropoda) y Chironomus sp. (Artropoda). El patrón de diversidad comunitario disminuyó en relación al incremento de la gradiente de contaminación minera existente en el lago. Pleuroxus caca Baird 1843, P. laevis Sars 1861, P. trigonellus (Müller 1785) y P. truncatus (Müller 1785) son nuevos registros para el Lago Junín. El nivel trófico predominante fueron los descomponedores. Se concluye que la evaluación rápida de biodiversidad (ERB) en la Reserva Nacional de Junín es relativamente alta para el zooplancton.
\end{abstract}

Palabras claves: cladocera, conservación, Lago Junín, Pleuroxus, rotífero

\begin{abstract}
Zooplankton was assessed from four localities of the Junín Natural Reserve, Peru,: Upamayo Bridge, Upamayo Reservoir, Pari and Ondores. Alpha and beta diversity for zooplanktonic communities were calculated. Zooplankton was assigned to trophic guilds. A total of 35 zooplanktonic taxa were found. The following is the descending sequence related to aquatic species richness: Pari $>$ Upamayo Reservoir $=$ Upamayo Bridge $>$ Ondores. Cladocerans were more abundant and with the highest species richness at Pari. Six species were shared by the four localities: Brachionus dimidiatus Bryce 1931 (Rotifera), Rhabdolaimus terrestris de Mann 1880 (Nematoda), Echiniscus sp. (Tardigrada), Aelosoma variegatum Vejdovsky 1866 (Annelida), a species of calanoid (Arthropoda) and Chironomus sp. (Arthropoda). Community diversity diminished in relation to increasing of the gradients mining contamination in the Junín Lake. Pleuroxus caca Baird 1843, P. laevis Sars 1861, P. trigonellus (Müller 1785) y P. truncatus (Müller 1785) are new records for the Lake. Decomposers were the predominant level. We conclude that rapid biodiversity assessment (RBA) at the Junín National Reserve is relatively high for zooplankton.
\end{abstract}

Key words: cladocera, conservation, Junin Lake, Pleuroxus, rotifer

\section{Introducción}

El lago Junín es uno de los cuerpos de agua más importantes dentro de la variedad geohidrológica en el Perú, debido a su extensión, ubicación y las fluctuaciones anuales del volumen hídrico de este ecosistema. Este recurso hídrico condiciona la formación de zonas especiales a nivel de la orilla, generando una gran variedad de hábitats y nichos ecológicos. De esta forma se suscita un entorno ecológico para una variedad de especies endémicas de aves, peces, anfibios anuros, y de modo especial para los organismos representantes del zooplancton que dependen directamente de estos cambios de nivel del recurso acuático, producido principalmente por las compuertas en la Represa de Upamayo (Alvariño, 1990; Valdivia \& Alvariño, 1991; Huaylinos \& Quispitupac, 2004). Sin embargo, está contaminado en una tercera parte por los relaves mineros provenientes de las minas cercanas, provenientes del río San Juan (Brack, 1986; Arrascue, 2001). Así, el estudio del zooplancton es de gran interés ya que hasta la fecha los trabajos realizados son pocos (Alvariño, 1990).

La evaluación del zooplancton es de fundamental importancia para el entendimiento de la transferencia energética en las comunidades continentales y en los ciclos biogeoquímicos (Prat et al., 1999; Metzeling et al., 2003; Silva et al., 2003). Los indicadores biológicos, entre ellos el zooplancton, son especies o 
un grupo taxonómico que con su presencia pueden mostrar el estado de la biota referente a parámetros como biodiversidad y biogreografía o grado de intervención humana (Peveling et al., 1999; Adams \& Greeley, 2000; Sánchez et al., 2001; Iannacone et al., 2002; Mac Nally \& Fleshmann, 2003; Kati et al., 2004).

Los objetivos del presente trabajo fueron evaluar el zooplancton en la Reserva de Junín: (1) en el Puente de Upamayo, (2) en la Represa de Upamayo, (3) en Pari y (4) en Óndores identificando los componentes zooplanctónicos y los niveles de similitud faunística entre las zonas muestreadas.

\section{Materiales y métodos}

Área de estudio

La Reserva Nacional de Junín se encuentra ubicada en los departamentos de Junín y Pasco Perú, al noreste en la Meseta de la Pampa de Junín a 4100

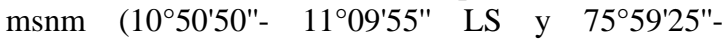
$\left.76^{\circ} 15^{\prime} 40^{\prime \prime} \mathrm{LW}\right)$, cubriendo una extensión de 53000 has. La extensión del lago es de 14300 has (Alvariño, 1990; Huaylinos \& Quispitupac, 2004).

El clima se caracteriza por presentar dos periodos bien diferenciados: periodo lluvioso (Octubre - Abril) y periodo seco (Mayo - Setiembre) (SEDAPAL, 1999). La precipitación anual oscila entre los 80 a 110 $\mathrm{mm}$ anuales, la temperatura promedio anual varia desde los $-2^{\circ} \mathrm{C}$ hasta $16^{\circ} \mathrm{C}$. El presente estudio se realizó durante el periodo seco, durante Setiembre de 2000.

Diversidad del Zooplancton

El estudio se realizó en cuatro localidades de la Reserva Nacional de Junín: (1) la Represa de Upamayo (15 de Septiembre, 3 puntos de muestreo y (2) el Puente de Upamayo (15 de Septiembre, 3 puntos de muestreo), (3) Pari (16 de Septiembre, 8 puntos de muestreo) y (4) Óndores (17 de Septiembre, 10 puntos de muestreo), departamento de Junín, provincia de Junín (Figura 1). Para la selección de los puntos de muestreo se tomó en cuenta las recomendaciones utilizadas por Valdivia \& Zambrano (1989). La caracterización de las cuatro localidades en zona litoral abierta, accesible y protegida siguió a lo descrito por Alvariño (1990). La Tabla 1, nos muestra las principales características de los puntos de muestreo donde se colectó el zooplancton en el ambiente acuático.

Análisis cualitativo: Para obtención de las muestras litorales se utilizó una red manual de plancton de $75 \mu$ de abertura de porosidad y $15 \mathrm{~cm}$ de diámetro (Wetzel \& Likens, 2000). Se pasó la red 30 veces a nivel de la superficie del agua y entre la vegetación macrofítica presente. El zooplancton concentrado en las redes fue vertido a frascos de boca ancha de $200 \mathrm{~mL}$ y posteriormente se les adicionó formol azucarado al 10 \%, para lograr una concentración de fijación y preservación al 4 \% (Valdivia \& Zambrano, 1989), luego fueron etiquetados y transportados al laboratorio para su posterior clasificación.

Análisis cuantitativo: Se procedió a filtrar el agua, teniendo cuidado con el volumen medido con la ayuda de un balde plástico de $4 \mathrm{~L}$, con una red manual de malla nytal ${ }^{\circledR}$ de $75 \mu$ de abertura de poro y $15 \mathrm{~cm}$ de diámetro. El volumen filtrado fue de $20 \mathrm{~L}$ (Keppeler \& Hardy, 2004). Se siguió el mismo procedimiento para la fijación y preservación indicada en el análisis cualitativo. Posteriormente fueron etiquetados y transportados al laboratorio para su posterior conteo e identificación taxonómica. En el análisis cuantitativo se procedió a medir el volumen total de la muestra en una probeta de $100 \mathrm{~mL}$, luego se tomaron submuestras de $3 \mathrm{~mL}$ y se colocaron en una placa Petri de plástico de $60 \mathrm{~mm}$ de diámetro para su observación al microscopio a $400 \mathrm{X}$ y $1000 \mathrm{X}$ de aumento, procediéndose luego al conteo de los especímenes girando la placa en zigzag. La densidad de organismos zooplanctónicos se presentó en $\mathrm{N}^{\circ}$ organismos $\cdot 20 \mathrm{~L}^{-1}$ (APHA, 1995).

Para la identificación de los especímenes se utilizaron los trabajos sobre cladóceros de la laguna de Paca y de lagunas altoandinas del Perú y de copépodos de vida libre en el Perú (Valdivia \& Zambrano, 1989; Del Río \& Valdivia, 1989; Alvariño, 1990; Valdivia \& Burger, 1990). Además de la colaboración de los profesionales especialistas en cada grupo respectivo.

\section{Análisis de Datos}

Se usó el coeficiente de correlación Spearman $\left(\mathrm{r}_{\mathrm{s}}\right)$ para determinar la relación entre las abundancias de especies del zooplancton entre las cuatro localidades evaluadas. Se empleó el estadístico no paramétrico de Friedman y la prueba de Kendall para determinar si existían diferencias significativas en las abundancias de las especies de zooplancton para las cuatro localidades censadas del Lago Junín. El nivel de significancia empleado fue de alfa $=0.05$ (Zar, 1996). Se utilizó el paquete estadístico SPSS versión 11.5 en español para el cálculo de los estadísticos descriptivos e inferenciales.

Para el sistema acuático se calculó la diversidad ecológica alfa mediante el índice de Shannon-Wiener (H’) (Favila \& Halffter, 1997; Camargo, 1999), donde $\mathrm{H}^{\prime}=-$ - piLnpi siendo $\mathrm{Ln}=$ logaritmo neperiano; $($ pi) $=$ ni $/ \mathrm{N}$, (ni) = número de individuos por cada especie o género, $(\mathrm{N}) 0$ número total de individuos. El otro índice utilizado fue el de Simpson (C), que varia entre 0 y 1 cuya fórmula es: $\mathrm{C}=1$ $\Sigma(n i / N)^{2}$ en el que " $n$ " representa el número de ejemplares de cada taxa y "N" el número total de ejemplares en la muestra, siendo el valor equivalente a 1 como el de máxima diversidad. Para el análisis de la diversidad ecológica beta comparativo de similaridad entre las cuatro localidades se aplicaron los índices 
cualitativos de Sörensen y de Kulcynski (Medri \& Lopes, 2001; Iannacone et al., 2002).

\section{Resultados}

Diversidad del zooplancton

Se encontraron un total de 35 morfotaxas (Tabla 2). Los artrópoda fueron los más númerosos $(n=22)$, luego los rotíferos con ocho especies, y con una especie cada una, los filas Rhizopoda, Cnidaria, Nematoda, Tardigrada y Annelida. Con relación a la riqueza de especies del zooplancton por localidad de muestreo, la secuencia en orden descendente fue: Pari $>$ Represa de Upamayo = Puente de Upamayo > Óndores. En cambio con relación al número de organismos zooplanctónicos $\cdot 20 \mathrm{~L}^{-1}$ fue en orden descendente: Pari > > Öndores $>$ Represa de Upamayo $>$ Puente de Upamayo. Siendo esto último corroborado al aplicarse la prueba no paramétrica de Friedman $\left(\chi^{2}=28.78\right.$; g.l. $=3$; $\left.\mathrm{P}=0.000\right)$ y la prueba de Kendall $(\mathrm{W}=0.274 ; \mathrm{P}=0.000)$, que indicó diferencias significativas en la abundancia de organismos zooplanctónicos entre Pari y las otras tres localidades del lago Junín.

Con relación al número de especies de rotífera, se presentó un total de 8 morfoespecies (Tabla 2); sin embargo al evaluarse el número de especies en relación con la localidad muestreada en orden descendente fue: Puente de Upamayo $(n=6)=$ Pari $(n=6)>$ Represa de Upamayo $(n=5)>$ Óndores $(n=$ 1). Los cladóceros presentaron un total de catorce especies, presentando por localidad el siguiente orden descendente: Pari $(\mathrm{n}=13)>$ Represa de Upamayo (n $=5)=$ Puente de Upamayo $(n=5)>$ Óndores $(n=2)$. Solo seis especies zooplanctónicas se encontraron presentes en las cuatro localidades: Brachionus dimidiatus (Rotifera), Rhabdolaimus terrestris (Nematoda), Echiniscus sp. (Tardigrada), Aelosoma variegatum (Annelidae), una especie no indentificada de calanoideo (Arthropoda) y formas larvarias de primer estadio de Chironomus sp. (Arthropoda). Además, 7 especies se encontraron en tres localidades, 6 en dos localidades y 16 especies solo fueron registradas en una sola localidad. Ningún cladócera se presentó en común a las cuatro localidades censadas. Con relación a los gremios alimentarios la secuencia fue: descomponedor $(n=25)>$ fitófago $(n=9)>$ depredador $(\mathrm{n}=1)$. Las tres especies más abundantes en cada una de las localidades fueron: para Represa de Upamayo: Keratella cochlearis (Rotifera) > Lecane lunaris (Rotifera) > B. dimidiatus (Rotifera); para Puente de Upamayo: K. cochlearis (Rotifera) $>R$. terrestris (Nematoda) > A. variegatum (Annelida); para Pari: A. variegatum (Annelida) > Trichocerca bicristata (Rotifera) > Lecane leontina (Rotifera) y finalmente para Óndores: Chironomus sp. (Arthropoda) $>$ R. terrestris (Nematoda) $>$ Amphipoda Talitridae (Arthropoda) (Tabla 2).
Los valores de temperatura del agua y del aire no presentaron diferencias estadísticamente significativas entre las cuatro localidades del lago Junín evaluadas para el zooplancton (Tabla 3).

Con relación a los índices de diversidad de Shannon-Wiener y de Simpson, los valores más altos fueron para Represa de Upamayo y para Pari; así como para Pari y Puente de Upamayo, respectivamente (Tabla 3). Se encontraron valores significativos en los coeficientes de correlación de Spearman, Kulcynski y Sörensen para Puente y Represa de Upamayo; así como para Represa de Upamayo y Óndores (Tablas 4 y 5).

\section{Discusión}

Valdivia \& Burger (1990) señalan que el estudio de las comunidades zooplanctónicas presentes en los ecosistemas altoandinos, como es el Lago Junín, es importante desde el punto de vista zoogeográfico y ecológico. Se han registrado para el Perú 105 especies de cladóceros, lo que sobrepasa a lo registrado en otros países Neotropicales. Sin embargo, es aún pobre en comparación a otras latitudes. En este estudio, se ha registrado en una sola localidad como es Pari, 13 especies de cladóceros (92.87 \% del total). Desde el punto de vista ecológico, estas especies dan valiosa información como comunidades indicadoras, ya que muchas especies son estenoicas (endémicas) y otras presentan una valencia ecológica alta (cosmopolitas) (Valdivia \& Burger, 1990).

A pesar de que Pari, se encuentra más cerca a las localidades de Represa de Upamayo y de Puente de Upamayo (junto a los flujos de contaminación por los ríos Colorado y San Juan) que Óndores, presenta una alta diversidad de zooplancton (Tabla 2), en términos de riqueza y abundancia de especies, principalmente cladóceros, quizás debido a la gran cantidad de vegetación presente en dicha zona, que protege de la contaminación del lago, como un colchón de soporte (Tabla 1), y a la mayor temperatura del agua (Tabla 3). Sin embargo, los puntos de muestreos en Óndores que presentaron poca diversidad, estuvieron asociados a un puquial con poca vegetación, en los que los pobladores realizaban lavado de ropa empleando detergente (Valdivia \& Alvariño, 1991). Valdivia \& Alvariño (op. cit.) señalan a Óndores como un lugar representativo del lago por la mayor diversidad, y a Pari con muy baja diversidad. En el presente estudio, más de 15 años después se encontró al parecer un patrón que podría ser opuesto (Tabla 4). Algunas especies endémicas de lagos altoandinas, no registradas anteriormente para el Lago Junín, como $P$. caca, P. laevis, $P$. trigonellus y $P$. truncatus han sido registrados por primera vez para el Lago Junín (Tabla 2). Este incremento en la dispersión en un tiempo relativamente corto en especies Pleuroxus ha sido registrado en otros cladóceros como Daphnia para 
cuerpos de agua dulceacuícolas en Argentina (Adamowicz et al., 2004).

Arrascue (2001) en una evaluación ecotoxicológica de los sedimentos de Puente de Upamayo en el lago Junín a una profundidad de $12 \mathrm{~m}$ encontró una alta contaminación por metales pesados, principalmente $\mathrm{Fe}, \mathrm{Cu}, \mathrm{Zn}$, Cd y $\mathrm{Pb}$, por lo que al usar a Chironomus calligraphus Goeldi a $48 \mathrm{~h}$ y a la bacteria Escherichia coli a 30 min de exposición durante Abril a Octubre del 2000, se registraron altos efectos ecotóxicos.

Fussmann (1996) ha señalado que la especie de rotífero Keratella cochlearis no incrementa poblacionalmente cuando los crustáceos cladóceros zooplantónicos están presentes debido a mecanismos competitivos, al alimentarse los cladóceros de sus recursos fitoplanctónicos. Angeler et al. (1999), Seda \& Devetter (2000) y Riofrío et al. (2003) indican que altas poblaciones de rotíferos zooplanctónicos, coinciden con reducidas abundancias de crustáceos zooplanctónicos procedentes de cuerpos de agua continentales. En este caso se observó que $K$. cochlearis presentó mayor abundancia en las localidades de Represa y Puente Upamayo, cuando la abundancia y riqueza de los crustáceos cladócera eran menores, en comparación con Pari, en la que se observa un patrón opuesto entre $K$. cochlearis y cladóceros (Tabla 2).

Modenutti (1998) señala una mayor diversidad del zooplancton en cuerpos lóticos (tipo ríos) en comparación a los ambientes lénticos (tipo lagunas). En el presente trabajo la localidad de Pari (ambiente léntico) parece ser que presentó una mayor diversidad zooplanctónica en comparación con Óndores (ambiente lótico) (Tabla 2). Keppeler \& Hardy (2004) encontraron que el rotífero loricado K. cochlearis en el Lago Amapá, en Brasil, fue componente dominante dentro del zooplancton. Esta especie presentó una alta abundancia (Tabla 2). Las especies del género Lecane han sido empleadas como bioindicadoras de toxicidad aguda para evaluar metales pesados (Pérez-Legaspi \& Rico-Martinez, 2001). Lecane leontina, es una especie que podría ser altamente dominante solo en la localidad de Pari, quizás debido a la vegetación presente en esta localidad que la protege de la contaminación (Tabla 2). Por otro lado, para evaluar la calidad de agua, son empleados comúnmente los quironómidos como indicadores biológicos (Iannacone et al., 2000).

\section{Agradecimientos}

A mis estudiantes del Curso de Conservación de Recursos Naturales de la Escuela Profesional de Biología, Semestre 2000 por su colaboración en el muestreo en la Reserva Nacional de Junín. Este trabajo fue presentado en representación de la Universidad Nacional Federico Villarreal en la XI Reunión Científica del Instituto de Investigación de
Ciencias Biológicas (ICBAR) de la Universidad Nacional Mayor de San Marcos del 24 al 26 de Abril del 2002.

\section{Literatura citada}

Adamowicz S.J., Hebert P.D.N. \& Marinote C. 2004. Species diversity and endemism in the Daphnia of Argentina: a genetic investigation. Zoological Journal of the Linnean Society. 140: 171-205.

Adams S.M. \& Greeley M.S. 2000. Ecotoxicological indicators of water quality. Using multi-response indicators to assess the health of aquatic ecosystems. Water, Air, and Soil Pollution. 123: 103-115.

Alvariño L.F. 1990. Fauna Cladócera (Crustacea) del Lago Junín. Tesis para optar el Título de Licenciado en Biología. Universidad Ricardo Palma. Facultad de Ciencias Biológicas. 169 pp + Anexos.

Angeler D.C., Alvarez M.C., Rojo C. \& Sánchez S.M. 1999. The significance of water inputs to plankton biomass and trophic relationships in a semi-arid freshwater wetland (central Spain). Journal of Plankton Research. 22: 2075-2093.

APHA (American Public Health Association), AWWA (American Water Works Association), WPCF (Water Pollution Control Federation). 1995. Standard methods for examination of water and wastewater. $19^{\text {th }} \mathrm{Ed}$. Washington, DC.

Arrascue A. 2001. Dos ensayos ecotoxicológicos para evaluar la calidad de sedimentos elutriados dulceacuícolas. Tesis para optar el Título de Licenciado en Biología. Universidad Nacional Federico Villarreal. Lima, Perú.

Borror D., de Long D. \& Thriplehorn C. 1995. An introduction to the study of insects. Saunders College Publishing. $6^{\text {th }}$ Ed. USA.

Brack E.A. 1986. Las ecorregiones del Perú. Boletín de Lima (Perú). 44: 57-70.

Camargo A. 1999. Estudo comparativo sobre a composicao e a diversidade de lepidopteros noturnos em cinco áreas de regiao dos cerrados. Revista brasileira de Zoologia. 16: 369-380.

Del Río M. \& Valdivia R. 1989. Copépodos dulceacuícolas de vida libre en el Perú. Familia Cyclopidae. Orden Cyclopoida. Boletín de Lima (Perú). 63: 71-80.

Favila M.E. \& Halffter G. 1997. The use of indicator groups for measuring biodiversity as related to community structure and function. Acta Zoológica Mexicana. (n.s.). 72: $1-25$.

Fussmann G. 1996. The importance of crustacean zooplankton in structuring rotifer and phytoplankton communities: an enclosure study. Journal of Plankton Research. 18: 1897-1915.

Huaylinos W.V. \& Quispitupac E.Q. 2004. Estado actual y efecto de la caza local de aves acuáticas en la Reserva Nacional de Junín. Dilloniana. 4: 81-82.

Iannacone J., Dale W. \& Alvariño L. 2000. Monitoreo ecotoxicológico del río Rímac (Lima- Perú) empleando a Chironomus calligraphus Goeldi (Diptera: Chironomidae). Revista Chilena de Entomología. 27: 23-34.

Iannacone J., Mansilla J. \& Ventura K. 2002. Macroinvertebrados en Las Lagunas de Puerto Viejo, Lima -Perú. Ecología Aplicada. 2: 116-124. 
Kati V., Devillers P., Dufrene M., Legakis A., Vokou D. \& Lebrun P. 2004. Testing the value of six taxonomic groups as biodiversity indicators at a local scale. Conservation Biology. 18: 667-675.

Keppeler E.C. \& Hardy E.R. 2004. Vertical distribution of zooplankton in the water column of Lago Amapá, Rio Branco, Acre, Brazil. Revista brasileira de Zoologia. 21: 169-177.

Mac Nally R. \& Fleishman E. 2004. A successful predictive model of species richness based on indicator species. Conservation Biology. 18: 646-654.

Medri I.M. \& Lopes J. 2001. Coleopterofauna em floresta e pastagem no norte do Paraná, Brasil, colectada con armadilha de solo. Revista brasileira de Zoologia. 18: 125-133.

Metzeling L., Chessman B., Hardwick R. \& Wong V. 2003. Rapid assessment of rivers using macroinvertebrates: the role of experience, and comparison with quantitative methods. Hydrobiology. 510: 39-52.

Modunetti B.E. 1998. Planktonic rotifers of Samborombón River Basin (Argentina). Hydrobiology. 387-388: 259265.

Pérez-Legaspi I.A. \& Rico-Martinez R. 2001. Acute toxicity on three species of the genus Lecane (Rotifera: Monogononta). Hydrobiology. 446-447: 375-381.

Peveling R., Rafanomezantsoa J.J., Razafinirina R., Tovonkery R. \& Zafimaniry G. 1999. Environmental impact of locust control agent fenitrothion, fenitrothionesfenvalerate and triflumuron on terrestrial arthropods in Madagascar. Crop Protection. 18: 659-676.

Prat N., Toja J., Sola C., Burgos M.D., Plans M. \& Rieradevall M. 1999. Effect of dumping and cleaning activities on the aquatic ecossytems of the Guadiamar River following a toxic flood. Science Total Environment. 242: 231-248.
Riofrío J., Samanez I., Carrasco F. \& Clavo M. 2003. Caracterización limnológica de la laguna de Cashibococha (Ucayali-Perú) durante el año 2001. Revista peruana de biología. 10: 183-194.

Sánchez C.V., Townsend A. \& Escalante P. 2001. El modelado de la distribución de especies y la conservación de la diversidad biológica. pp. 359-379. En: Enfoques contemporáneos para el estudio de la biodiversidad. H. Hernández; A.M. García; F. Álvarez \& M. Ulloa (Eds.). Instituto de Biología. UNAM. México.

Seda J. \& Devetter M. 2000. Zooplankton community structure along a trophic gradient in a canyon-shaped reservoir. Journal of Plankton Research. 22: 1829-1840.

SEDAPAL. 1999. El País de las lagunas. Historia y Ecología de la Puna de Junín. Sedapal. Taller Gráfica Biblos. Lima, Perú.

Silva T., Neumann-Lietao S., Schwamborn R., Gusmao O. \& Nascimento-Viera D.A. 2003. Diel and seasonal changes in the macrozooplankton community of a tropical estuary in Northeasthern Brazil. Revista brasileira de Zoologia. 20: 439-446.

Valdivia R. \& Alvariño L. 1991. El Lago Junín: Un Recurso natural en contaminación progresiva. Boletín de Lima (Perú). 76: 35-38.

Valdivia R. \& Burger L. 1990. Observaciones sobre la fauna Cladócera de algunas lagunas Alto andinas del Perú. Boletín de Lima (Perú). 71: 63-79.

Valdivia R. \& Zambrano F. 1989. Cladóceros de la Laguna de Paca, Junín. Relaciones ecológicas entre hábitat y especie. Boletín de Lima (Perú). 64: 83-89.

Wetzel R.G. \& Likens G.E. 2000. Limnological analyses. $3^{\text {th }}$ Ed. Springer-Verlag. New York.

Zar H. 1996. Biostatistical Analysis. $3^{\text {th }}$ Ed. Prentice-Hall. Inc. Upper Saddle River. New Yersey.

\section{Tablas citadas en el texto}

Tabla 1. Principales características de las cuatro localidades de colecta de zooplancton del Lago de Junín.

\begin{tabular}{|c|c|}
\hline Localidades de muestreo & Características principales de las localidades de muestreo \\
\hline $\begin{array}{l}\text { Represa de Upamayo } \\
\text { (tres puntos de colecta) }\end{array}$ & $\begin{array}{l}\text { Los tres puntos presentaron características semejantes entre sí. Zona Litoral Abierta. Caracterizada porque la } \\
\text { orilla no está ocupada por plantas. Las olas alcanzan libremente la orilla. El fondo frecuentemente es } \\
\text { arenoso o pedregoso. Este litoral no está aislado en ningún momento de la influencia pelagial. Ambiente } \\
\text { léntico. }\end{array}$ \\
\hline $\begin{array}{l}\text { Puente de Upamayo } \\
\text { (tres puntos de colecta) }\end{array}$ & $\begin{array}{l}\text { Los tres puntos presentaron características semejantes entre sí. Zona Litoral Accesible. Este tipo de litoral } \\
\text { está ocupado generalmente por totorales, que no crecen densamente y por lo tanto atenúan los movimientos } \\
\text { de las olas. Las olas alcanzan la orilla. Este tipo de litoral tiene una barrera segura al alcance de la influencia } \\
\text { de la zona pelagial. Ambiente léntico. }\end{array}$ \\
\hline $\begin{array}{l}\text { Pari } \\
\text { (ocho puntos de colecta) }\end{array}$ & $\begin{array}{l}\text { Punto 1: Zona litoral cerrada. Presenta abundante materia orgánica y poca cantidad de algas macrofíticas; se } \\
\text { encuentra completamente aislado de la influencia de la zona pelagial y de poca profundidad. } \\
\text { Punto 2: Zona litoral cerrada. Agua con abundante turbidez, presencia de fango y se encuentra } \\
\text { completamente aislada de la zona pelagial, de poca profundidad. } \\
\text { Punto 3: Zona litoral accesible. Abundancia de alga macrofíticas y poca turbidez del agua con poca } \\
\text { influencia pelagial y con una profundidad considerable. } \\
\text { Punto 4: Zona litoral cerrada. Zona fangosa, con abundante materia orgánica en descomposición y se } \\
\text { encuentra completamente aislada de la zona pelagial, de poca profundidad. } \\
\text { Punto 5: Zona litoral cerrada. Zona con abundante vegetación macrofítica, agua con mucha claridad y de } \\
\text { poca profundidad. } \\
\text { Punto 6: Zona litoral cerrada. Zona fangosa con abundante materia orgánica en descomposición, deshechos } \\
\text { de basura y evidente influencia antropogénica, masa de agua de poca profundidad. } \\
\text { Punto 7: Zona litoral accesible. Abundante vegetación macrofítica, de poca profundidad y con poca } \\
\text { influencia pelagial. } \\
\text { Punto 8: Zona litoral cerrada. Totalmente aislado de la zona pelagial, con abundante materia orgánica en } \\
\text { descomposición y agua con bastante turbidez y de poca profundidad. } \\
\text { Todos los puntos fueron ambientes lénticos. }\end{array}$ \\
\hline $\begin{array}{l}\text { Óndores } \\
\text { (10 puntos de colecta) }\end{array}$ & $\begin{array}{l}\text { Zona litoral accesible. Los } 10 \text { puntos fueron tomados de las aguas procedentes de un puquial -riachuelo que } \\
\text { desemboca finalmente en el lago Junín. Aguas con escasa materia orgánica, poca profundidad y poca } \\
\text { vegetación macrofítica. Puntos separados por } 5 \text { m entre sí. Ambientes lóticos. }\end{array}$ \\
\hline
\end{tabular}


DIVERSIDAD DEL ZOOPLANCTON EN LA RESERVA NACIONAL DE JUNÍN, PERÚ Diciembre 2006

Tabla 2. Comunidades Zooplanctónicas en el Lago Junín, Septiembre del 2000.

\begin{tabular}{|c|c|c|c|c|c|}
\hline Phylum & Represa Upamayo & Puente Upamayo & Pari & Óndores & Posición trófica \\
\hline \multicolumn{6}{|l|}{ Rhizopoda } \\
\hline Centropyxis aculeata (Ehrenberg, 1832) & - & 0.333 & - & - & D \\
\hline \multicolumn{6}{|l|}{ Cnidaria } \\
\hline Chlorohydra viridissima (Pallas, 1766) & - & 0.333 & 4.750 & - & $\mathrm{P}$ \\
\hline \multicolumn{6}{|l|}{ Rotifera } \\
\hline Brachionus dimidiatus Bryce, 1931 & 1 & 0.333 & 54.250 & 0.100 & $\mathrm{D}$ \\
\hline Keratella cochlearis (Gosse, 1851) & 4 & 2.333 & 0.125 & - & D \\
\hline Lecane leontina (Turner, 1892) & - & 0.333 & 58.250 & - & D \\
\hline Lecane lunaris (Ehrenberg, 1832) & 1.333 & 0.666 & & - & D \\
\hline $\begin{array}{l}\text { Lecane monostyla quadridentata (Daday, } \\
\text { 1897) }\end{array}$ & - & - & 0.375 & - & $\mathrm{D}$ \\
\hline Philodina acuticornis Murray, 1902 & - & 0.333 & - & - & D \\
\hline Platyias quadricornis (Enrenberg, 1834) & - & - & 0.125 & - & D \\
\hline Trichocerca bicristata (Gosse, 1887) & 0.333 & 0.666 & 81,500 & - & D \\
\hline \multicolumn{6}{|l|}{ Nematoda } \\
\hline Rhabdolaimus terrestris de Mann, 1880 & 0.666 & 1 & 198 & 2.900 & $\mathrm{D}$ \\
\hline \multicolumn{6}{|l|}{ Tardigrada } \\
\hline Echiniscus sp. & 0.333 & 0.333 & 53.750 & 0.200 & F \\
\hline \multicolumn{6}{|l|}{ Annelida } \\
\hline Aelosoma variegatum Vejdovsky, 1866 & 0.333 & 1 & 144.500 & 1.500 & D \\
\hline \multicolumn{6}{|l|}{ Artropoda } \\
\hline $\begin{array}{l}\text { Alona cambouei Guerne \& Richard, } \\
\text { 1893* }\end{array}$ & 0.333 & 0.333 & 8.250 & - & D \\
\hline Alona costata Sars, $1862 *$ & - & - & 9.250 & - & D \\
\hline Alona sp.* & - & - & 0.125 & - & $\mathrm{D}$ \\
\hline Pleuroxus aduncus (Jurine, 1820)* & 0.333 & - & 10 & 0.100 & $\mathrm{D}$ \\
\hline Pleuroxus caca Baird, 1843* & 0.333 & 0.333 & 18.500 & - & $\mathrm{D}$ \\
\hline Pleuroxus laevis Sars, 1861* & - & - & 0.125 & - & $\mathrm{D}$ \\
\hline Pleuroxus trigonellus (Müller, 1785)* & - & - & 6 & - & $\mathrm{D}$ \\
\hline Pleuroxus truncatus (Müller, 1785)* & - & - & 38 & - & $\mathrm{D}$ \\
\hline Acroperus harpae (Baird, 1834)* & - & - & 16.500 & - & $\mathrm{D}$ \\
\hline Camptocercus similis Sars, 1901* & - & - & 0.250 & - & $\mathrm{D}$ \\
\hline Chydorus globosus (Baird, 1843)* & - & - & 10.750 & - & D \\
\hline Daphnia peruviana Harding, 1955* & - & - & 18 & - & $\mathrm{F}$ \\
\hline Ceriodaphnia dubia Richard, 1894* & 0.666 & 0.333 & - & 0.200 & $\mathrm{~F}$ \\
\hline Simocephalus vetulus (Müller, 1776)* & 0.333 & - & 4 & - & $\mathrm{F}$ \\
\hline Ostrácoda sp. no identificada & - & - & 48.250 & 0.800 & D \\
\hline $\begin{array}{l}\text { Acarina hydrachnnelidae sp. no } \\
\text { identificada }\end{array}$ & 0.333 & - & - & - & $\mathrm{D}$ \\
\hline Paracyclops fimbriatus (Fisher, 1853) & 0.333 & - & - & 0.400 & $\mathrm{~F}$ \\
\hline Harpaticoidea sp. no identificada & 0.666 & 0.333 & - & 0.200 & $\mathrm{~F}$ \\
\hline Calanoidea sp. no identificada & 0.666 & 0.666 & 6.250 & 0.300 & $\mathrm{~F}$ \\
\hline Nauplios de Copépoda & - & - & 3.250 & - & $\mathrm{F}$ \\
\hline Chironomus sp. (larvas) & 0.333 & 0.333 & 2.500 & 7,800 & $\mathrm{D}$ \\
\hline Amphipoda Talitridae sp. no identificada & - & - & - & 1.700 & $\mathrm{~F}$ \\
\hline Organismos $\cdot 20 \mathrm{~L}^{-1}$ & 12.333 & 10 & 795.62 & 16.2 & \\
\hline Total de morfotaxas & 18 & 18 & 27 & 12 & \\
\hline
\end{tabular}


Tabla 3. Temperatura y de diversidad de comunidades zooplanctónicas en cuatro localidades del Lago Junín.

\begin{tabular}{|c|c|c|c|c|}
\hline Temperatura & $\begin{array}{c}\text { Represa de } \\
\text { Upamayo } \\
\end{array}$ & $\begin{array}{l}\text { Puente de } \\
\text { Upamayo } \\
\end{array}$ & Pari & Óndores \\
\hline Agua & $\begin{array}{c}10.52 \pm 2.13 \\
(8.5-12)\end{array}$ & $\begin{array}{c}10.94 \pm 1.77 \\
(9-12.5)\end{array}$ & $\begin{array}{c}14.95 \pm 2.19 \\
(11-18)\end{array}$ & $\begin{array}{c}8.85 \pm 1.24 \\
(10-13)\end{array}$ \\
\hline Aire & $\begin{array}{c}12.7 \pm 1.89 \\
(10-14.2)\end{array}$ & $\begin{array}{c}12.5 \pm 1.66 \\
(9.8-13.8)\end{array}$ & $\begin{array}{c}11.17 \pm 1.69 \\
(9-13.40)\end{array}$ & $\begin{array}{c}11.40 \pm 0.96 \\
(10-13)\end{array}$ \\
\hline Diversidad de Shannon- Wiener $\left(\mathrm{H}^{\prime}\right)$ & $1.63 \pm 1.83$ & $1.29 \pm 0.71$ & $1.57 \pm 0.41$ & $1.18 \pm 0.79$ \\
\hline Diversidad de Simpson (C') & $0.48 \pm 0.37$ & $0.64 \pm 0.21$ & $0.76 \pm 0.07$ & $0.48 \pm 0.29$ \\
\hline Número de puntos Muestreados & 3 & 3 & 8 & 10 \\
\hline
\end{tabular}

Tabla 4. Coeficiente de correlación de Spearman de las abundancias de las comunidades zooplanctónicas de cuatro localidades del Lago Junín.

Coeficiente de Correlación de Spearman

\begin{tabular}{cccccc} 
& & \multicolumn{3}{c}{ Coeficiente de Correlación de Spearman } \\
\cline { 3 - 6 } & Localidades & $\begin{array}{c}\text { Represa de } \\
\text { Upamayo }\end{array}$ & $\begin{array}{c}\text { Puente de } \\
\text { Upamayo }\end{array}$ & Pari & Óndores \\
\cline { 2 - 6 } Probabilidad & Represa de Upamayo & - & $\underline{0.66}$ & 0.03 & $\underline{0.42}$ \\
& Puente de Upamayo & $\underline{0.00}$ & - & 0,18 & 0,27 \\
& Pari & 0.85 & 0.29 & - & 0.11 \\
\hline & Óndores & $\underline{0.01}$ & 0.11 & 0.51 & - \\
\hline
\end{tabular}

Tabla 5. Índices de similaridad de Kulcynski y Sörensen de comunidades zooplanctónicas de cuatro localidades del Lago Junín.

\begin{tabular}{cccccc} 
& \multicolumn{4}{c}{ Índice de Similaridad de Kulcynski } \\
\hline & Localidades & $\begin{array}{c}\text { Represa de } \\
\text { Upamayo }\end{array}$ & $\begin{array}{c}\text { Puente de } \\
\text { Upamayo }\end{array}$ & Pari & Óndores \\
\cline { 2 - 6 } Índice de & Represa de Upamayo & - & $\underline{1.00}$ & 0.19 & $\underline{0.59}$ \\
Sörensen & Puente de Upamayo & $\underline{1.00}$ & - & 0.19 & 0.21 \\
& Pari & 0.38 & 0.38 & - & 0.00 \\
\hline
\end{tabular}

\footnotetext{
${ }^{1}$ Laboratorio de Ecofisiología Animal. Facultad de Ciencias Naturales y Matemáticas. Universidad Nacional Federico Villarreal. Calle San Marcos 383, Pueblo Libre, Lima 21, Perú.

${ }^{2}$ E-mail: joseiannacone@hotmail.com
} 